\title{
Revisión de la implementación de Lean Six Sigma en Instituciones de Educación Superior
}

\author{
Implementation of Lean Six Sigma in Higher Education Institutions: \\ A comprehensive review
}

\author{
David Rodrigo Guerrero Moreno ${ }^{1 *} \quad$ Jorge Antonio Silva Leal ${ }^{1}$ \\ Claudia Cristina Bocanegra-Herrera ${ }^{2}$
}

Recibido 27 de abril de 2018, Aceptado 13 de septiembre de 2018

Received: April 18, 2017 Accepted: September 13, 2018

\begin{abstract}
RESUMEN
Las metodologías de Lean Manufacturing, Six Sigma y Lean Six Sigma (LSS) han sido ampliamente implementadas en diversos ámbitos, escenarios e industrias; las Instituciones de Educación Superior (IES) no han sido la excepción; sin embargo, su implementación ha tenido y tiene barreras por superar. El propósito de este artículo es presentar un análisis de diversos escritos de estudio de casos en los que se ha implementado Six Sigma, Lean Manufacturing o su integración LSS en IES y de artículos conceptuales con el fin de examinar la manera en que se han aplicado estas metodologías en IES, sus motivaciones y principios necesarios requeridos para una efectiva implementación.

La revisión muestra que se han efectuado diversos proyectos de mejora con estas metodologías en procesos académicos, de currículo, de servicios y administrativos. Se encontró que entre los motivadores más importantes que tienen las IES para implementar estas metodologías se encuentran, entre los más relevantes: reducir costos de la gestión de calidad educativa, mejorar el sistema de calidad y acreditación de la institución educativa, responder a la alta competencia, mejorar eficiencia y efectividad en los procesos académicos y administrativos, incrementar la satisfacción de partes interesadas y responder a la reducción de presupuestos en las IES. La implementación de LSS es factible siempre y cuando se instauren principios como el liderazgo, visión, cultura de cambio, entrenamiento, adecuada selección de proyectos, medición de los procesos para toma de decisiones y compromiso de la alta dirección.
\end{abstract}

Palabras clave: Six Sigma, Lean Manufacturing, Lean Six Sigma, instituciones de educación superior, motivadores, principios.

\begin{abstract}
The methodologies of Lean Manufacturing, Six Sigma and Lean Six Sigma (LSS) have been widely implemented in different industries, and higher education institutions (HEIs) have not been the exception; however, its implementation has had barriers to overcome.

The purpose of this article is to present a review of case study articles of Six Sigma, Lean Manufacturing or LSS implementation in HEIS and conceptual articles in order to analyze how these methodologies have been implemented in HEIs, motivators, and principles necessary for effective implementation.

The review shows that improvement projects are implemented with these methodologies in academic, curriculum, service, and administrative processes. It was found that among the motivators most important
\end{abstract}

1 Facultad de Ingeniería. Universidad Santiago de Cali. Calle 5 \# 62-00. Cali, Colombia.

E-mail: david.guerrero01@usc.edu.co; jorge.silva04@usc.edu.co

2 Facultad de Ingeniería. Universidad Nacional de Colombia. Carrera 45 Nº 26-85. Bogotá, Colombia.

E-mail: cbocanegra@unal.edu.co

* Autor de correspondencia: david.guerrero01@usc.edu.co 
are to reduce educational quality management costs; to improve the quality and accreditation system of the university; to respond to high competition; to improve the efficiency and effectiveness of academic and administrative processes; to increase stakeholder satisfaction, and to respond to budget reduction to universities.

It is feasible to implement LSS if some principles are applied such as leadership and vision, the culture of change, training, adequate selection of projects, measurement of processes for decision-making, and commitment of senior management.

Keywords: Six Sigma, Lean Manufacturing, Lean Six Sigma, higher education institutions.

\section{INTRODUCCIÓN}

En el marco del mejoramiento continuo, las organizaciones a través del tiempo han mejorado sus prácticas de calidad, la exigencia de los mercados y de los clientes hizo que se pensara la calidad de una forma estratégica [1]. Con ello surgieron prácticas de aseguramiento de calidad y calidad total (TQM) que han generado grandes beneficios a distintos tipos de organizaciones, alcanzando altos niveles de desempeño y satisfacción del cliente [2-3]. En este marco, se han generado distintas metodologías modernas como lo son Six Sigma y Lean Manufacturing que se han incorporado a la estrategia organizacional en empresas del sector público y privado; en grandes, medianas y pequeñas empresas obteniendo grandes beneficios medidos en términos de eficiencia y efectividad [4-5].

Six Sigma tiene por objeto reducir la variabilidad de los procesos y con ello disminuir el número de defectos, entendiendo por estos algo que produce insatisfacción en los clientes, mientras que Lean Manufacturing busca eliminar desperdicios en los procesos [6]. A su vez, la metodología integrada Lean Six Sigma (LSS) se centra en la eliminación de desperdicios, reducción de la variación para lograr la satisfacción del cliente y la mejora financiera en los resultados del negocio con respecto a la calidad, la entrega y el costo [7]. Ambas metodologías fueron creadas en ambientes manufactureros; sin embargo, en los últimos años estas metodologías han sobrepasado fronteras de diferentes industrias alcanzando excelentes resultados [8-9].

Las Instituciones de Educación Superior (IES), tienen actualmente la necesidad de obtener procesos más efectivos, eficientes y económicos; para ello, han buscado y deben encontrar distintas y diversas metodologías que aseguren estos resultados [10].
Además, las IES juegan un papel muy importante en la sociedad en razón a que generan beneficios para las ciencias y la tecnología [9]. En los últimos años las IES se han apoyado en metodologías de mejora de la calidad como TQM [2]; sin embargo, algunas instituciones han ido mucho más allá implementando proyectos de Six Sigma con el objetivo de mejorar el desempeño académico de sus estudiantes [11-12], mejorar servicios ofrecidos a los mismos [13] o mejorar procesos académicos y de currículo $[1,14]$.

De análoga manera, proyectos de Lean Manufacturing que se han enfocado en la mejora de diseños de curso y currículo y mejora de procesos han resultado eficaces en este objetivo. Se resalta el caso de Winona State University que ha usado esta filosofía de forma masiva en diferentes procesos como contratación, tecnología de información y acreditación de programas [15]. Algunos de los beneficios que se han obtenido son: proporcionar un enfoque de resolución de problemas, hacer más visibles los procesos, obtener información sobre la voz del cliente interno y externo, cumplir eficazmente requisitos de acreditación de calidad, promover la participación de equipos en proyectos de mejora, establecer indicadores efectivos e identificar y reducir costos ocultos [16].

En los últimos años las IES se han volcado a buscar acreditaciones internacionales de alta calidad de sus programas, lo cual se constituye en un proceso de reconocimiento de programas educativos en el desempeño, integridad y calidad, brindando, de esta manera, confianza a la comunidad educativa lo que redunda en legitimidad y posicionamiento académico nacional e internacional, dado el origen del reconocimiento, ejemplo "ABET" es una organización no lucrativa y no gubernamental, considerada como autoridad reconocida a nivel internacional en el tema de acreditación de programas de ciencias aplicadas, informática, ingeniería y tecnología [17]. 
La implementación de estas metodologías en IES ha encontrado varios obstáculos, barreras y retos que han propiciado un ritmo lento para la misma, debido a que, por obvias razones, no es lo mismo ponerlas en práctica en el sector manufacturero que en el sector educativo [18]. Estas barreras han sido identificadas y definidas por varios autores en la literatura del tema de calidad. A título de ejemplo podemos tipificar algunas situaciones conocidas en este entorno: el papel que tienen los estudiantes como cliente, pero también como producto de un servicio; diversos indicadores de calidad en procesos administrativos y académicos; dificultad en la obtención y análisis de datos; presencia de factores no cuantificables e incontrolables; falta de pensamiento orientado y basado en procesos; falta de visión estratégica; falta de comunicación, cultura y recursos, entre otros $[4,19,21]$.

Estas barreras pueden ser superadas si se aplican principios en las IES que aseguren una implementación y sostenibilidad de LSS. Autores como Lu et al. [18] hablaban de la necesidad de realizar una exploración de los modelos de liderazgo en las IES con el fin de definir unos principios de liderazgo, los cuales fueron identificados y definidos desde el punto de vista de aquellos; sin embargo, se considera necesario hacer una investigación sistemática, más profunda para el establecimiento de unos principios generales en la implementación de LSS en IES. Además, hasta el momento no se ha realizado una revisión de motivos que tienen las IES para implementar Lean Manufacturing, Six Sigma o LSS, lo que genera un valor agregado a la presente investigación.

\section{ANTECEDENTES DE LEAN MANUFACTURING Y SIX SIGMA EN IES}

Se hizo consulta de artículos en las bases de datos: "Scopus", "Emerald", "Proquest", "Science Direct" e "Engineering Village" del que resultaron 49 artículos distribuidos en artículos conceptuales, estudio de casos, modelos de implementación y un solo artículo de revisión de las metodologías Six Sigma, Lean Manufacturing y LSS en IES, como lo muestran la Figura 1 y Figura 2.

Se puede observar que la producción de artículos en las metodologías presentadas se ha realizado en igual proporción, sin embargo, debido a la integración de la metodología Lean Manufacturing y Six Sigma, la producción en LSS en IES inicio en gran medida desde el año 2014; desde el 2014 a 2018 se ha producido el $90 \%$ de artículos en esta metodología.

Se observa además que aproximadamente la mitad de los artículos son estudio de casos; mientras que existe una baja producción de artículos que definen "modelos de implementación" de alguna de estas metodologías. Cabe resaltar que no se encontró ningún artículo que establezca un modelo de aplicación de la metodología de Lean Manufacturing en IES, en la literatura se encuentran modelos genéricos de implementación de las diferentes metodologías de mejora continua [22]. Sin embargo, hacen falta modelos metodológicos de implementación diseñados para aplicar en una industria en especial.

La Figura 3 evidencia que la producción de artículos ha tenido un crecimiento importante desde el año

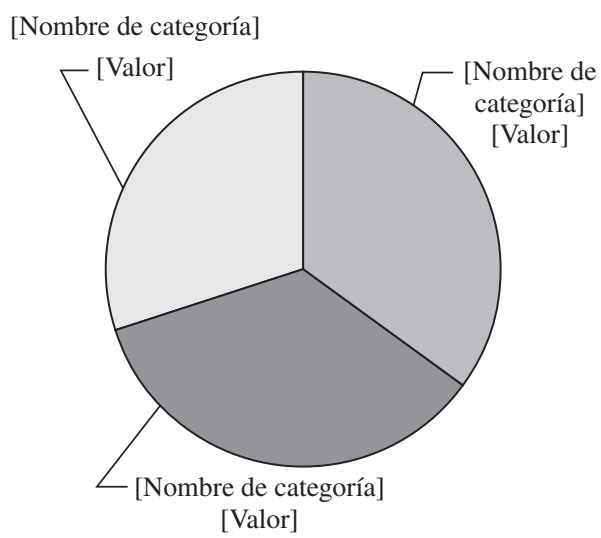

Figura 1. Producción de artículos por metodología.

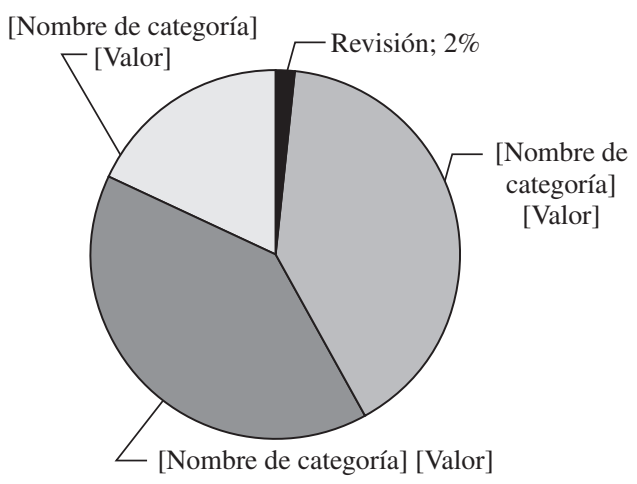

Figura 2. Producción de artículos por tipo de contribución. 


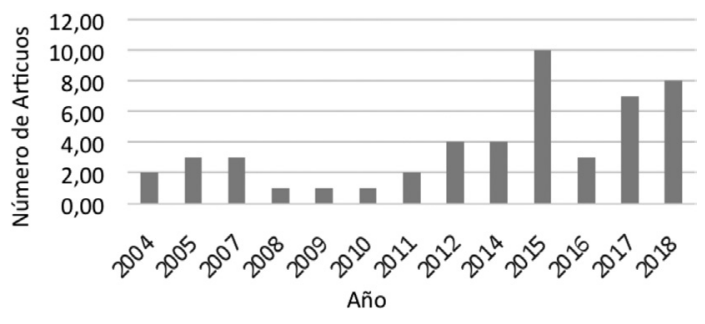

Figura 3. Número de artículos por año de Six Sigma, Lean Manufacturing y Lean Six Sigma en IES.

2010, alcanzó su punto más alto en el año 2015, con el $20 \%$ del total de la producción de artículos; presentó una caída en el año 2016 y tuvo un crecimiento importante en el año 2017. En el año 2018 se presenta una producción alta de artículos, que si continúa con esta tendencia, se mostraría como el año de mayor producción de investigación de estas metodologías en IES. Los países que han generado la mayor contribución de artículos son: Estados Unidos (39\%) y Reino Unido (29) y las revistas que más tienen artículos son "International Journal of Productivity and Performance Management" (20\%) y "International Journal of Quality \& Reliability Management" (16\%).

\section{SIX SIGMA EN IES}

Six Sigma es una metodología estructurada, organizada para reducir variación, aumentar el desempeño en procesos organizacionales y lograr altos niveles de calidad cumpliendo con objetivos estratégicos valiéndose de especialistas de mejora [23]. El concepto de Six Sigma fue introducido por Motorola en la década de 1980 para mejorar sus productos y mantener la calidad de los mismos; desde entonces, Six Sigma ha sido exitosamente utilizada e implementada en diferentes industrias [8].

Las IES demuestran sus propias características operacionales que definen el propio sector de servicios donde operan. Las IES enfrentan desafíos especiales para la implementación de Six Sigma; sin embargo, esta metodología puede resultar efectiva en resolver los problemas en procesos propios de una institución educativa con muchas y diversas soluciones a los mismo, siempre y cuando sea tomada como una estrategia de la organización [4]. Jenicke et al. [19] discuten la naturaleza única de Six Sigma en la IES y en su estudio descriptivo encuentran que, si bien son únicas, las IES pueden adoptar un marco estratégico de Six Sigma alrededor de los diferentes clientes que tiene este tipo de organizaciones (estudiantes, directivos de facultad o colaboradores administrativos). Definir al cliente desde el punto de vista de la IES es difícil por la variedad de partes interesadas, los entornos que habitan este tipo de clientes dan como resultado entornos de trabajo significativamente diferentes [19].

Algunas IES ya han implementado proyectos de Six Sigma. El primer ejemplo, cronológicamente hablando, lo constituye la Universidad Mercer en Atlanta, Estados Unidos, donde se implementó la metodología en la Facultad de Ingeniería para integrar tres enfoques diferentes: el sistema métrico, la comunicación constante y el cambio cultural [24]. Cabe resaltar la situación que ocurrió por los años 2007 a 2009 en las IES de este país, que enfrentaron, en plena crisis económica, una reducción de presupuestos importante, bajo la continua presión del gobierno, lo que propició que varias de ellas se apoyaron en la implementación de Six Sigma [25] obteniendo ahorros importantes.

Consecuentemente con lo anotado, Bandyopadhyay [26] desarrolla un modelo para el enfoque de Six Sigma, el cual define las actividades a desarrollar y las herramientas a aplicar en cada etapa del ciclo DefinirMedir-Analizar- Mejorar y Controlar (DMAIC) para perfeccionar la calidad y productividad en una IES. El modelo resultante presenta un diagrama para el proceso de diseño de un programa educativo y utiliza como herramienta principal la matriz de despliegue de funciones QFD que involucra a las partes interesadas (agentes acreditadores, posibles empleadores, padres, etc.). Por medio de una encuesta de identificación de necesidades y expectativas se realiza un diseño preliminar del programa ofrecido a un mercado de prueba, perfeccionando los programas mediante ciclos repetidos de rediseño y pruebas de campo para finalmente ofrecer los programas con una campaña de mercadeo adecuado.

Revisando la literatura se constata que se han desarrollado diferentes proyectos de Six Sigma enfocados al mejoramiento de procesos académicos o al mejoramiento de servicios propios para los estudiantes. En cuanto al mejoramiento de procesos académicos se encuentra el caso de la Universidad de 
Texas en el que se utilizaron equipos de estudiantes y ayudas tecnológicas para el proceso de aprobación del currículo y de curso con el fin de reducir el tiempo de ciclo, logrando una reducción del 78.9\% [27]. Otro ejemplo en mejora de procesos académicos lo constituye el caso aplicado por Kukreja et al. [28] en el cual implementaron Six Sigma para mejorar el curriculo del programa de Administración de Empresas con el fin de obtener la acreditación del programa. A pesar de encontrar grandes ventajas en su implementación, el autor observó que se requiere y se consume un tiempo importante en la implementación de esta metodología, especialmente en la obtención de los datos. Six Sigma también ha sido implementado para reducir el número de estudiantes que reprueban un curso [12]. Mazumder [11] en el 2014 establece que los principios de Six Sigma de mejoramiento de procesos, reducción de desperdicio y mejoramiento continuo se alinean en gran medida con la misión de IES así como los propósitos de acreditación de alta calidad.

Respecto al mejoramiento de servicios a estudiantes, Ramasubramanian [10] describe la implementación de Six Sigma en una institución técnica de educación para mejorar el desempeño de las operaciones desde el proceso de selección de estudiantes hasta su proceso de graduación. El resultado más importante lo constituye la definición de clientes en una IES y las recomendaciones que da el autor, antes de introducir Six Sigma en la IES. Entre las más relevantes se encuentran: organizar un equipo de trabajo y establecer la visión de gestión a largo plazo; provisionar primero la educación Six Sigma a la alta dirección; seleccionar un área piloto y establecer mensualmente el día Six Sigma en el que se verificará por la alta dirección el progreso de Six Sigma.

González [29] desarrolla un modelo de implementación de Six Sigma en IES en el que sugiere una serie de reactivos e indicadores para que sea una iniciativa de éxito en las IES mexicanas. El propósito del modelo es ofrecer una ventaja competitiva para elevar la calidad educativa; lograr su adaptación a los cambios y demandas del entorno y lograr los mejores beneficios para la sociedad mexicana.

En la Universidad Francisco de Paula Santander, experimenta la implementación de un proyecto Six Sigma, en la que se evidenciaron problemas de gestión organizacional y de centralización del proceso para la toma efectiva de decisiones. Una vez se implementó el proyecto se observó mejoras en la capacidad de procesos; efectividad del 99,9\% en variables de medición; mejora en percepciones de la comunidad académica y administrativa y estandarización de proceso [1].

Como se refirió anteriormente, Six Sigma tiene el propósito de reducir la variación y con ello reducir defectos, por lo cual se revisaron los avances sobre la definición del concepto de defecto en IES. Varios autores han aportado ejemplos de lo que constituye defecto en una IES como lo muestra la Tabla 1.

\section{LEAN MANUFACTURING EN IES}

Lean Manufacturing se originó como una filosofía de simplificación de los procesos y eliminación de

Tabla 1. Tipos de defectos en IES.

\begin{tabular}{|l|c|}
\hline \multicolumn{1}{|c|}{ Defecto } & Autores \\
\hline $\begin{array}{l}\text { Reprobaciones de exámenes. Se refiere a la incapacidad de no lograr un mínimo puntaje en una escala } \\
\text { de valoración para aprobar un examen. }\end{array}$ & {$[12]$} \\
\hline $\begin{array}{l}\text { Reprobación de curso. Se refiere a la incapacidad de no lograr un mínimo puntaje en una escala de } \\
\text { valoración para aprobar un curso. }\end{array}$ & {$[11]$} \\
\hline Deserción de estudiantes. Se refiere al estudiante que abandona una institución de educación superior [30] & {$[31]$} \\
\hline Incumplimiento de metas de indicadores en procesos académicos. & {$[1]$} \\
\hline Servicios que sobrepasen un tiempo estándar. & {$[9]$} \\
\hline $\begin{array}{l}\text { Falta de formación y sensibilización de personal. } \\
\text { Diferencia en los tiempos de respuesta de admisión. } \\
\text { Tiempos muertos internos en el procedimiento. } \\
\text { Descentralización del proceso. }\end{array}$ & {$[13]$} \\
\hline
\end{tabular}


desperdicios en la producción de bienes y prestación de servicios, entendiendo estos como tiempos de ciclos largos e innecesarios; tiempos de espera entre actividades que no generan valor [32] [3]. Lean Manufacturing no es un concepto estático, su novedad consiste en la combinación de distintos elementos, técnicas y herramientas surgidas para mejoramiento del flujo de producción, apoyadas por la alta dirección en pleno convencimiento de su necesidad [33].

El desperdicio se define como cualquier actividad humana que absorbe recursos, pero no crea valor [34]; sin embargo, es necesario revisar este concepto en las IES [35]. Diferentes autores ya han dado sus aportes en su definición, algunos ejemplos de desperdicios en IES se resumen en la Tabla 2.

Las IES comenzaron a implementar Lean Manufacturing desde el año 2000 en niveles de departamento, área y nivel organizacional [40]. En el nivel de área y departamento, Emiliani [41] mejora el diseño de cursos y currículo en programas de maestría de Gestión y Administración de Empresas usando Lean Manufacturing. En un primer estudio de caso se evidenciaron mejoras en el marco de la acreditación de calidad en un curso de la maestría de administración en la escuela de negocios de Rensselaer Polytechnic Institute. Los resultados basados en encuestas formales anónimas y retroalimentación informal a mitad de semestre indicaron que la satisfacción de los estudiantes mejoró sustancialmente. Se hallaron diferentes desperdicios que se eliminaron con herramientas propias de Lean Manufacturing, por ejemplo, "5S" para organizar contenido curricular, dar secuencia al curso y eliminar material que no generaba valor; "Just in Time" para la entrega de proyectos y trabajos calificados a tiempo para discusión en próxima clase; estandarización de formatos de plan de curso; rubricas de evaluación; controles visuales para mostrar errores comunes en que incurren los estudiantes, con detrimento en sus calificaciones, usando diferentes colores en la evaluación de trabajos del curso y hojas de cálculo que permiten mostrar, a simple vista, el desempeño de los estudiantes.

En un segundo caso aplicado en esta misma institución con el fin de mejorar el currículo de los diez (10) cursos que conformaban el programa de Maestría en Administración para ejecutivos se utilizó la técnica "Kaizen". Se crearon equipos "Kaizen" que estaban conformados por estudiantes (voz del cliente), profesores de cada curso, miembros del programa y facilitadores. El evento "Kaizen" tenía una duración de uno (1) o dos (2) días para la búsqueda de la mejora y se realizaba un evento por curso. Entre los resultados más importantes que se lograron están: la definición de objetivos y propósitos de los cursos; actualización de material de cursos y aplicación de conceptos en casos reales de negocios [42].

Es importante analizar el caso de Winona State University, la cual usó Lean para mejorar más de 40 procesos operacionales y de apoyo que incluían la acreditación del programa de enfermería. Se encontró que uno de los factores de éxito más importante es la composición de equipos que trabajan los proyectos de mejora. Después de ejecutar diferentes proyectos se llegó a la conclusión consistente en que se requiere un tiempo considerable para coordinar, facilitar e implementar las mejoras. La solución a esta circunstancia podría ser brindar entrenamiento y liderazgo desde la alta dirección [15].

Se observa que proyectos aplicados con la metodología Lean Manufacturing se limitaron a mejoras que afectaron a un pequeño porcentaje de estudiantes, profesores o empleados, razón por lo cual, se sugirió que un proyecto con un impacto en toda la universidad conllevaría a un mejor uso de los recursos [15].

IES de varios países han experimentado la implementación de Lean; los pioneros en Estados Unidos son: University of Central Oklahoma, University of Iowa, University of New Orleans, Bowling Green State University, University of Scranton, Rensselaer Polytechnic Institute y WSU $[21,37]$. En Europa, la Universidad de Cardiff ubicada en Gales [43] y la Universidad de St Andrews, de Escocia [44], lideraron este proceso; sin embargo, estas IES representan una pequeña muestra de instituciones que vieron en Lean una opción de mejora viable para la educación superior.

Un caso especial en el que se combina Lean Manufacturing con otra metodología de gestión como lo es Gestión de Relaciones con el Cliente (CRM) lo constituye la Pontificia Universidad JaverianaBogotá en donde se aplicó esta metodología en el programa de Ingeniería Industrial como insumo de 
Tabla 2. Tipos de desperdicios en IES.

\begin{tabular}{|c|c|c|}
\hline $\begin{array}{c}\text { Tipo } \\
\text { Desperdicio }\end{array}$ & Ejemplo & Autores \\
\hline \multirow{6}{*}{ Procesamiento } & Múltiples aprobaciones y controles en un proceso & {$[35$} \\
\hline & Ayuda excesiva al estudiante & [36] \\
\hline & $\begin{array}{l}\text { Horarios diseñados de acuerdo a preferencias de profesores y directores y no } \\
\text { de los estudiantes. } \\
\text { Pasos de un proceso inspeccionados en exceso porque no son confiados a la } \\
\text { primera vez. } \\
\text { Cambio de información de reportes y datos en diferentes formatos. }\end{array}$ & [37] \\
\hline & Ubicación de libros en los puestos asignados de una biblioteca. & [9] \\
\hline & $\begin{array}{l}\text { Duplicación de información. } \\
\text { Copias extras. }\end{array}$ & [38] \\
\hline & Contenido innecesario de cursos. & [39] \\
\hline \multirow{5}{*}{ Sobreproducción } & Carga de trabajo desequilibrada en semestres y programación desigual. & [35] \\
\hline & Servicios desarrollados para un cliente no especificado. & [38] \\
\hline & Temas ya enseñados en otros cursos o temas obsoletos. & [36] \\
\hline & $\begin{array}{l}\text { Evaluaciones de desempeño realizadas únicamente por cumplir con requerimientos. } \\
\text { Documentación en ubicaciones centrales y en facultades. } \\
\text { Información irrelevante que resulta en decisiones y acciones inapropiadas. } \\
\text { Perdida de folletos de publicidad de programas debido a cambios en el mismo. }\end{array}$ & [37] \\
\hline & $\begin{array}{l}\text { Clases sin su capacidad de estudiantes. } \\
\text { Mas graduados de los requeridos en el mercado laboral. }\end{array}$ & [39] \\
\hline \multirow{3}{*}{ Inventario } & Más suministros o artículos de lo requerido. & [35] \\
\hline & $\begin{array}{l}\text { Hojas de vida de profesores en diferentes lugares para diferentes propósitos. } \\
\text { Espacios físicos y edificios usados ineficientemente. } \\
\text { Personal, equipos e instalaciones sobrecargadas o subutilizadas. } \\
\text { Igual cantidad de personal en épocas de vacaciones de estudiantes. }\end{array}$ & [37] \\
\hline & Ingreso de mismos datos en repetidas ocasiones. & [38] \\
\hline \multirow{4}{*}{ Espera } & $\begin{array}{l}\text { Largas colas de espera para acceder a servicios. } \\
\text { Largas esperas para aprobación de documentos. } \\
\text { Caída en los sistemas de información. } \\
\text { Búsqueda extensa de archivos, libros y documentos. } \\
\text { Tiempos de respuesta de solicitudes extensos. }\end{array}$ & [35] \\
\hline & $\begin{array}{l}\text { Tiempos de espera por inicio de reuniones, proyectos o informes para ser completados. } \\
\text { Tiempos de espera para nivelación de estudiantes. }\end{array}$ & [36] \\
\hline & Procedimientos poco claros que generan espera. & [38] \\
\hline & $\begin{array}{l}\text { Tiempos de espera para inscribirse en un curso. } \\
\text { Tiempos de espera para inicio de clase. }\end{array}$ & [39] \\
\hline \multirow{4}{*}{ Movimientos } & $\begin{array}{l}\text { Movimientos innecesarios de estudiantes y colaboradores entre una área o } \\
\text { departamento para acceder a un servicio. }\end{array}$ & [35] \\
\hline & Modificación de la oferta de profesores por sobrecupo en cursos o falta de inscritos. & [37] \\
\hline & Movimientos innecesarios para búsqueda de libros en biblioteca. & [9] \\
\hline & Excesivo envío de datos e información. & [38] \\
\hline \multirow{4}{*}{ Defectos } & Errores en la introducción de datos. & [35] \\
\hline & Prestación de un servicio a un ritmo lento y con errores. & [37] \\
\hline & Documentación incompleta. & [38] \\
\hline & No desarrollar competencias en un curso. & [39] \\
\hline \multirow{3}{*}{ Transporte } & Múltiples aprobaciones. & [35] \\
\hline & $\begin{array}{l}\text { Espacios de almacenamiento en ubicaciones remotas. } \\
\text { Transporte innecesario de materiales, información y personas. }\end{array}$ & $\begin{array}{l}{[37]} \\
{[38]}\end{array}$ \\
\hline & Aprobaciones innecesarias. & [38] \\
\hline
\end{tabular}


Lean para la definición de valor agregado requerido por los usuarios de este programa [45].

La implementación de Lean Manufacturing ofrece una oportunidad para que las IES mejoren sus procesos académicos y administrativos y, a su vez, su competitividad en el mercado. La aplicación de Lean y otras estrategias de reducción de costos tienen un enorme potencial en el sector educativo para la mejora de la calidad y la sostenibilidad [40].

\section{LEAN SIX SIGMA EN IES}

La evolución de LSS empezó desde el año 2000 en que por primera vez se utilizó este término para describir un sistema que combina Lean Manufacturing y Six Sigma [46]. Una de las definiciones encontradas en la literatura sobre LSS establece que es una metodología que se enfoca en la eliminación de desperdicio y reducción de variación apoyándose en la estructura DMAIC con el fin de lograr satisfacción del cliente teniendo en cuenta la calidad, costo y entrega [46].

Autores como Snee [8] le dan a LSS una definición con mayor rango en los niveles organizacionales pues la define como una estrategia de negocio que incrementa el rendimiento de procesos y desarrolla satisfacción del cliente, liderazgo y resultados financieros que mejora la calidad, la eficiencia y los costos.

Como se mostró anteriormente, varias IES se han embarcado en la implementación de Lean o Six Sigma; sin embargo, pocas lo han hecho integrando Lean Six Sigma pese a la aplicabilidad y potenciales beneficios que puede dar la metodología. Cudney et al. [47] realizan una revisión sistemática de Lean Manufacturing y Six Sigma en IES para identificar las oportunidades existentes en este sector para estas metodologías, encuentran que la implementación es apropiada para mejorar métodos de enseñanza, procesos administrativos y otras facetas de las IES. Sunder [48] y Antony et al. [4] identificaron entre las principales barreras para la implementación de LSS las perspectivas de mercado para las IES, definición de cliente, detección de defecto, falta de uniformidad en estudiantes, sistema de medición y procesos operados por personas y para personas, lo que hace que exista una gran variabilidad.

No obstante, las IES tienen un gran potencial de implementar LSS, debido a que la gran mayoría de estas organizaciones definen la calidad como un foco estratégico dentro de su visión. Hess y Benjamin [49] identificaron áreas potenciales para la implementación de LSS en IES como son diseño de currículo, servicios administrativos, admisiones, inscripciones e, inclusive, investigación. Específicamente, estos autores establecen que LSS podría ser ideal para:

- Definir competencias y metodologías de aprendizaje del estudiante para el buen desarrollo del plan de estudios;

- Analizar y medir las variaciones de rendimiento y deserción de los estudiantes;

- Asignar recursos de programas académicos de una manera productiva;

- Definir políticas de pago y tarifas de matrículas;

- Analizar estadísticamente el consumo de energía en campus para identificar aquellas áreas donde se pueden obtener eficiencias;

- Analizar las variaciones en los costos de compra en contratos de servicio, suministro y materiales, así como identificar en que contratos podrían generarse ahorros;

- Analizar el flujo de trabajo en departamentos del campus para determinar número de personal necesario y así reducir costos.

Como se observa la implementación de LSS tiene un abanico amplio de oportunidades y obstáculos importantes en las IES que para ser gestionadas y superadas se requieren de un liderazgo significativo e importante en la alta dirección. En concordancia con lo anterior, Lu et al. [18] desarrolló un modelo de liderazgo de LSS que provee las características claves de liderazgo y establece que aquel puede ser la base para transformar a una institución de educación superior que se adapta a los cambios del contexto en el que la satisfacción del cliente y la eficiencia de los procesos son cruciales.

Ya se han realizado diferentes proyectos en IES en las áreas identificadas como ideales para la implementación de la metodología integrada Lean Six Sigma. Es el caso de una IES en el Reino Unido, donde se implementaron siete proyectos diferentes con diferentes propósitos como la reducción de cheques en el área financiera; disminución de tiempos en la compra y realización de software requerido en la IES; disminución de número de cambios de curso; reducción de número de reuniones y sus tiempos en el Comité de Información la institución; 
entre otros. Con la ejecución de estos proyectos se logró más transparencia en los procesos, reducción de costos y tiempos de procesos, reducción de tareas duplicadas entre departamentos, mejora en la moral de empleados y mayor conciencia por lograr excelencia en procesos [50].

Resulta interesante nombrar el proyecto que se ejecutó en una IES no especificada para reducir la deserción estudiantil utilizando la metodología Lean Six Sigma con la estructura DMAIC, se demostró que resulta viable para este tipo de problemas aplicar esta metodología, sin embargo, al ser un primer proyecto de este tipo se presentan barreras importantes por superar [51].

Con el fin de establecer un marco de referencia para la implementación de LSS en IES y aumentar el número instituciones que se embarcan en estas iniciativas de mejora, Antony et al. [52] desarrolla el primer modelo de aplicación que contiene siete etapas necesarias para una exitosa implementación de la metodología en una IES así como las herramientas que se deben utilizar en un proyecto de mejora LSS. Las siete etapas identificadas son: preparación de Lean Six Sigma, establecimiento de una necesidad, desarrollo de estrategia LSS, educación, formación, identificación de proyectos LSS y cierre de proyectos.

\section{MOTIVADORES PARA LA IMPLEMENTACIÓN DE LSS EN IES}

Se encontraron 15 motivadores o razones para implementar Lean Manufacturing, Six Sigma o LSS.

La Tabla 3 muestra la consolidación de los motivadores, identificando el autor o autores que lo describieron, así como la frecuencia de repetición. En general, y en su mayoría, las IES implementan una de estas dos metodologías (Lean Manufacturing y Six Sigma) o su integración porque desean:

Reducir costos de la gestión de calidad educativa. Las IES cada vez más buscan metodologías para

Tabla 3. Motivadores o razones para la implementación de LSS en IES.

\begin{tabular}{|r|l|l|l|}
\hline $\mathbf{N}^{\mathbf{o}}$ & \multicolumn{1}{|c|}{ Motivadores } & \multicolumn{1}{|c|}{ Autor(ES) } & Frecuencia \\
\hline 1 & $\begin{array}{l}\text { Reducir costos de la gestión de calidad } \\
\text { educativa }\end{array}$ & $\begin{array}{l}{[53],[54],[18],[55],[48],[20],[56],[38],[13],} \\
{[57],[58],[25],[28],[14],[40],[59],[47],[50],} \\
{[60],[61],[62]}\end{array}$ & 21 \\
\hline 2 & $\begin{array}{l}\text { Mejorar el sistema de calidad y } \\
\text { acreditación de las IES }\end{array}$ & $\begin{array}{l}{[54],[9],[48],[63],[64],[56],[38],[10],[57],} \\
{[26],[59],[52],[47],[65],[61],[62]}\end{array}$ & 16 \\
\hline 3 & $\begin{array}{l}\text { Mejorar eficiencia y efectividad de } \\
\text { procesos }\end{array}$ & $\begin{array}{l}{[18],[48],[1],[66],[20],[4],[10],[38],[13],} \\
{[26],[50],[60],[65],[61],}\end{array}$ & 14 \\
\hline 4 & $\begin{array}{l}\text { Responder a la alta competencia del } \\
\text { sector educativo }\end{array}$ & $\begin{array}{l}{[53],[55],[1],[20],[56],[38],[13],[10],[58],} \\
{[40],[59],[65],[61]}\end{array}$ & 13 \\
\hline 5 & $\begin{array}{l}\text { Mejorar procesos administrativos y } \\
\text { académicos }\end{array}$ & $\begin{array}{l}{[53],[1],[20],[64],[38],[57],[28],[29],[59],} \\
{[47],[60],[61]}\end{array}$ & 12 \\
\hline 6 & $\begin{array}{l}\text { Incrementar la satisfacción de partes } \\
\text { interesadas (estudiantes, docentes, } \\
\text { empleados y comunidad) }\end{array}$ & $\begin{array}{l}{[18],[48],[20],[11],[56],[4],[57],[29],[59],} \\
{[51],[52]}\end{array}$ & 11 \\
\hline 7 & $\begin{array}{l}\text { Responder a la reducción de presu- } \\
\text { puestos a universidades }\end{array}$ & $\begin{array}{l}{[25],[11][54],[18],[55],[11],[58],[26],[14],} \\
{[60],[62]}\end{array}$ & 11 \\
\hline 8 & $\begin{array}{l}\text { Cumplir metas e indicadores opera- } \\
\text { tivos y financieros }\end{array}$ & {$[18],[53],[9],[48],[4],[51],[52]$,} \\
\hline 9 & Reducir desperdicios & {$[18],[38],[26],[52],[50],[60],[61]$} & 7 \\
\hline 10 & Mejorar resultados académicos & {$[18],[53],[48],[28]$} & 7 \\
\hline 11 & Mejorar la clasificación de universidades & {$[40],[65],[61]$} & 4 \\
\hline 12 & Innovar procesos & {$[48],[61]$} & 3 \\
\hline 13 & Solucionar problemas & {$[53],[52]$} & 2 \\
\hline 14 & Reducir variación en procesos & {$[11]$,} & 1 \\
\hline 15 & Alinear procesos a objetivos estratégicos & {$[66]$} & 2 \\
\hline
\end{tabular}


mejorar la experiencia del estudiante que a su vez reduzcan desperdicio y costo $[38,55]$. LSS es una metodología de mejoramiento continuo atractiva para las IES porque al implementarla reduce costos de no calidad, mejora indicadores financieros y crea valor para clientes y partes interesadas [20]. Aunque la universidad es diferente de otras empresas en muchos aspectos, requiere una buena relación de costo-beneficio [57, 59].

Mejorar el sistema de calidad y acreditación de las IES. Las organizaciones LSS crean un ambiente de mejora continua a través del alineamiento de la organización al modelo; la metodología es más que una técnica de mejora de la calidad, es un enfoque de cambio que integra la capacidad de liderazgo con el mejoramiento de calidad, refinamiento de procesos y voz del cliente [9]. Estas metodologías han sido vistas como una ayuda adicional para responder a requisitos de acreditación por parte de los entes acreditadores del país donde operan [26, 64].

Mejorar eficiencia y efectividad de procesos administrativos y académicos. Con el entorno cambiante en la educación superior, donde la eficiencia y la efectividad se vuelven más imperativas debido a las crecientes restricciones presupuestarias y la alta competencia, los equipos de trabajo Lean pueden desempeñar un papel importante en la mejora de los procesos académicos y administrativos [58]. La enseñanza implica múltiples procesos, como el diseño de plan del curso, desarrollo curricular, instrucción en el aula, ejercicios de laboratorio, evaluación de aprendizaje del estudiante, etc., la metodología Six Sigma puede ser utilizada en todos los procesos mencionados [10].

Responder a la alta competencia del sector educativo. Día a día la competencia en el sector educativo se está incrementando, por lo cual la calidad de la educación será de primordial importancia en los programas ofrecidos [10]. Para encontrar éxito las IES deben ofrecer lo que otras instituciones no ofrecen, logrando una ventaja competitiva. Uno de los caminos más eficaces para este logro es usar los recursos de una manera eficiente reduciendo desperdicios en las diferentes actividades operacionales para de esta manera mejorar la calidad del servicio [38].

Incrementar la satisfacción de partes interesadas (estudiantes, docentes, empleados y comunidad).
La metodología Lean Manufacturing puede generar educación de alta calidad a bajos costos, con procesos eficientes que infunden orgullo, maximizan valor y tienen en cuenta los intereses de estudiantes y empleados en general [56]. Un enfoque integrado parece ser el mejor camino a seguir para las IES en lugar de confiar en Lean o Six Sigma para crear y mejorar el valor para los clientes y las partes interesadas [20].

Responder a la reducción de presupuestos en universidades. Las IES se enfrentaron a la reducción de presupuestos en Estados Unidos, India y Gran Bretaña. Se constataron casos que para responder a esta situación se implementó Lean Manufacturing, Six Sigma o LSS [26], [53], [55]; el resultado de esta decisión constituye un esfuerzo concertado de las instituciones académicas para reducir sus costos. Los esfuerzos de reducción de costos han tenido un efecto ostensible en las universidades públicas que han reducido las plantas de personal en todos los niveles [54].

Se observa que los motivadores o razones de implementación, con mayor frecuencia son motivadores de nivel estratégico; sin embargo, existen motivadores de nivel técnico que son propios de estas metodologías que no tuvieron una frecuencia importante como son la reducción de desperdicios, reducción de variación o solución de problemas de calidad.

\section{PRINCIPIOS PARA LA IMPLEMENTACIÓN DE LSS EN IES}

Así mismo, existen principios para la implementación de LSS, entre los que se encuentran:

En la Tabla 4 se identifica los principios para implementación, el autor o autores que lo describieron y su frecuencia de repetición. Los principios de mayor relevancia que son requeridos para una implementación efectiva y sostenible de LSS en IES son:

Liderazgo y visión. El rol activo del liderazgo ha sido ampliamente citado como uno de los factores esenciales de preparación para cualquier iniciativa de mejoramiento continuo en cualquier entorno organizacional. Los líderes deben establecer una visión clara para establecer la cultura deseada, permitir que los empleados pasen de sus prácticas 
Tabla 4. Principios de la implementación de LSS en IES.

\begin{tabular}{|c|c|c|c|}
\hline $\mathbf{N}^{\mathbf{o}}$ & Principios & Autor(es) & Frecuencia \\
\hline 1 & Liderazgo y visión. & $\begin{array}{l}{[21],[55],[20],[15],[67],[4],[10],[58],[25],} \\
{[29],[40],[52],[50],}\end{array}$ & 13 \\
\hline 2 & $\begin{array}{l}\text { Compromiso de la alta dirección y } \\
\text { disponibilidad de recursos. }\end{array}$ & [21], [55], [20], [66], [15], [67], [4], [52], [60], [65] & 10 \\
\hline 3 & Cultura de cambio. & {$[18],[55],[20],[4],[21],[63],[58],[25],[50]$} & 9 \\
\hline 4 & Entrenamiento. & {$[21],[20],[66],[15],[4],[10],[58],[25],[65]$} & 9 \\
\hline 5 & Adecuada selección de proyectos. & [9], [11], [63], [66], [15], [4], [10], [50], [60] & 9 \\
\hline 6 & $\begin{array}{l}\text { Medición de los procesos para toma } \\
\text { de decisiones. }\end{array}$ & {$[18],[20],[11],[58],[25],[29],[14]$} & 7 \\
\hline 7 & $\begin{array}{l}\text { Empoderamiento de los colaboradores } \\
\text { (profesores y administrativos) y trabajo } \\
\text { en equipo. }\end{array}$ & [18], [20], [49], [58], [25], [29], [50] & 7 \\
\hline 8 & $\begin{array}{l}\text { Enlazando LSS a la estrategia de la } \\
\text { universidad. }\end{array}$ & {$[21],[20],[63],[67],[14],[52]$} & 5 \\
\hline 9 & Efectiva comunicación. & [4], [58], [25], [40], [50] & 5 \\
\hline 10 & Adecuada selección de los colaboradores. & {$[21],[67],[52],[60]$} & 4 \\
\hline 11 & Enfoque al cliente. & [20], [49], [67], [25], & 4 \\
\hline 12 & $\begin{array}{l}\text { Estructura organizacional para operación } \\
\text { de LSS. }\end{array}$ & {$[21],[20],[60],[65]$} & 4 \\
\hline 13 & Incentivos, reconocimiento y motivación. & {$[18],[21],[65]$} & 3 \\
\hline 14 & Innovación para la mejora. & {$[54],[4]$} & 2 \\
\hline 15 & Implementación de proyectos piloto. & [21], [67] & 2 \\
\hline 16 & Equipo de “champions" y expertos. & {$[15],[60]$} & 2 \\
\hline 17 & Completar proyectos a tiempo. & [29] & 1 \\
\hline 18 & $\begin{array}{l}\text { Alineamiento de metas estratégicas con } \\
\text { las de facultad. }\end{array}$ & [11] & 1 \\
\hline
\end{tabular}

de trabajo actuales a prácticas de excelencia [40], [67], [68]. En el estudio realizado por Waterbury [15] se entrevistó a líderes de 7 universidades diferentes que implementaron Lean en sus instituciones, la necesidad de liderazgo fue mencionada en repetidas ocasiones como un factor crítico de éxito, donde el tiempo y los recursos invertidos en la implementación son fundamentales. Balzer et al. [21] establecen que se necesita liderazgo en tres actividades clave: en la concientización, en el entendimiento por parte de los colaboradores de la metodología y en la implementación de la misma.

Compromiso de la alta dirección y disponibilidad de recursos. El compromiso de la alta dirección se debe dar con la disponibilidad de recursos humanos y tecnológicos que permitan incrementar el desempeño de procesos [55]. Es indispensable la disponibilidad de tiempo para construir el talento humano mediante entrenamiento de herramientas propias de Lean Six Sigma, estadística y gestión de proyectos y brindar el tiempo necesario para la selección y ejecución de los mismos [4].

Cultura de cambio. Una de las principales dificultades para implementar mejoras de calidad en un entorno educativo superior es que la cultura académica a menudo no es receptiva. Algunas causas y/o razones en relación con lo anterior es la libertad académica que los profesores tienen en la enseñanza o lo que comúnmente se conoce como libertad de cátedra y las herramientas de modificación del comportamiento (como los aumentos salariales o las amenazas de despido) que son inaccesibles para los directores académicos [63]. Además, la cultura de la educación superior que ha resultado en universidades y facultades que se encuentran entre las instituciones más antiguas en el mundo, es duradera y difícil de cambiar [21], razón por la cual es necesario que la cultura de cambio sea 
desarrollada en los colaboradores. La cultura Lean debe ser el corazón de todo lo que se haga en la organización, los colaboradores deben ser educados y formados para que ellos con su pleno convencimiento y autónomamente generen la cultura Lean [18].

Entrenamiento. Con el fin de implementar efectivamente LSS, Svensson et al. [66] ilustran el caso de King Abdullah University of Science and Technology, donde se realizó un programa agresivo de entrenamiento que incluyó la preparación de 350 colaboradores de la parte administrativa y la certificación de 50 Yellow Belts, 150 Green Belts y siete Black Belts. Los autores concluyen que el programa LSS seguirá siendo la piedra angular para proporcionar al personal de la universidad las herramientas, el entrenamiento y la plataforma necesaria para la efectividad de LSS. La implementación exitosa de LSS únicamente puede darse si y solo si se da entrenamiento a colaboradores, el cual tiene un doble efecto, se da plataforma para ejecutar proyectos de mejora y cambia la cultura [25].

Adecuada selección de proyectos. Con el fin de mostrar las bondades que tiene LSS, se debe evaluar y seleccionar apropiadamente los proyectos de mejora a implementar; estos deberán tener un alto impacto en estudiantes y empleados [29]. Se han definido algunos criterios de selección: el proyecto debe estar alineado con las metas estratégicas de la organización; debe ser completado entre cuatro y seis meses; debe estar enfocado a mejorar una o dos métricas; debe mejorar percepciones del cliente sobre la calidad $o$ tener beneficios financieros $y$, finalmente, debe tener un apoyo total de la alta dirección [63]. De esta manera, la selección de proyectos deberá seguir una metodología que permita a las IES manejar grandes volúmenes de proyectos, comparar diferentes tipos de los mismos y pronosticar cuál de ellos producirá el mejor retorno [4].

Medición de los procesos para toma de decisiones. Las decisiones que se tomen en los procesos de las organizaciones deben ser sistemáticas y basada en datos reales [6]; sin embargo, la toma de datos en las IES ha sido considerada como una barrera por su inexistencia o por la dificultad de conseguirla. Antony [20] en su estudio en el que entrevistó a diferentes académicos destacados y expertos en LSS, coincidían en afirmar que obtener datos de variables cualitativas que son en su mayoría las variables que se presentan en IES representa una gran dificultad y que aún no se ha definido una metodología para análisis de datos en este tipo de organizaciones.

Los principios que no tuvieron una frecuencia alta pero que su aplicación es de gran importancia y que está relacionada con el liderazgo y compromiso de la alta dirección son: el enlace de LSS a la estrategia de la organización, la efectiva comunicación y la estructura organizacional para operación de LSS.

\section{CONCLUSIONES}

En los últimos años, las metodologías de Lean Manufacturing, Six Sigma y LSS se han implementado en IES en procesos misionales y de apoyo por diferentes causas, razones o motivadores. Se encontró que los motivos más importantes de implementación de estas metodologías han sido para: reducir costos de la gestión de calidad educativa; mejorar el sistema de calidad y acreditación de la universidad; responder a la alta competencia; mejorar eficiencia y efectividad de procesos académicos y administrativos; incrementar la satisfacción de partes interesadas y responder a reducción de presupuestos.

Entre los estudios de caso se observa que los beneficios más importantes son la mejora de la eficiencia de procesos académicos y administrativos, mejora de servicios, mejora de diseño de cursos y currículo, acreditación de programas y reducción de desperdicios. Estos últimos tienen una definición especial a la que varios autores se han referido, tratando de ejemplificar este concepto en IES.

Las IES que aplicaron Lean Manufacturing, Six Sigma y LSS no se han apoyado en estas metodologías, en gran medida en razón a que se encuentran con una serie de obstáculos en la adaptación al sector educativo. Entre las barreras más importantes que se han identificado están los diferentes roles que tienen los estudiantes considerado como cliente y producto; los diferentes indicadores de calidad en procesos administrativos y académicos; la dificultad en la obtención y análisis de datos; la presencia de factores no cuantificables e incontrolables; la falta de pensamiento basado en procesos; la falta de visión estratégica; la falta de comunicación, cultura y recursos, entre otras. 
Para sobreponerse a estas barreras y de esta manera implementar LSS de una forma sostenible, es necesario que las IES instituyan y se apropien de unos principios o factores críticos de éxito, como son conceptualizados en la literatura, entre los que se encuentran, de manera preponderante el liderazgo y visión, la cultura de cambio, el entrenamiento, la adecuada selección de proyectos, la medición de procesos para la toma de decisiones y el compromiso de la alta dirección y la disponibilidad de recursos de todo tipo.

\section{REFERENCIAS}

[1] M. Vergel-Ortega. "Filosofía gerencial seis sigma en instituciones universitarias". FaceRevista la Fac. Ciencias Econ. y Empres., pp. 99-106. 2015. ISSN: 1794-9920.

[2] M.B. Sirvanci. "Critical issues for TQM implementation in higher education". TQM Mag. Vol. $16 \mathrm{~N}^{\circ}$ 6, pp. 382386. 2004. ISSN: 0954-478X. DOI: 10.1108/09544780410563293.

[3] R. Andersson, H. Eriksson, H. Torstensson, R. Andersson, H. Eriksson and H. Torstensson. "Similarities and differences between TQM, six sigma and lean". TQM Mag. Vol. $18 \mathrm{~N}^{\circ} 3$, pp. 282-296. 2006. ISSN: 0954-478X. DOI: $10.1108 / 09544780610660004$.

[4] J.Antony, N. Krishan, D. Cullen and M. Kumar. "Lean Six Sigma for higher education institutions (HEIs)" Int. J. Product. Perform. Manag. Vol. 61 No 8, pp. 940-948. 2012. ISSN: 1741-0401. DOI: $10.1108 / 17410401211277165$.

[5] H. Felizzola Jiménez and C. Luna Amaya. "Lean Six Sigma en pequeñas y medianas empresas: un enfoque metodológico". Ingeniare. Rev. Chil. Ing. Vol. $22 \mathrm{~N}^{\mathrm{o}} 2$, pp. 263-277. 2014. ISSN: 0718-3305. DOI: 10.4067/S0718-33052014000200012.

[6] M.P.J. Pepper and T.A. Spedding. "The evolution of lean Six Sigma". Int. J. Qual. Reliab. Manag. Vol. $27 \mathrm{~N}^{\circ}$ 2, pp. 138155. 2010. ISSN: 0265-671X. DOI: $10.1108 / 02656711011014276$.

[7] R.J. Hilton and A. Sohal. "A conceptual model for the successful deployment of Lean Six Sigma". Int. J. Qual. Reliab. Manag. Vol. 29 $\mathrm{N}^{\circ} 1$, pp. 54-70. 2012. ISSN: 0265-671X. DOI: $10.1108 / 02656711211190873$.

[8] R.D. Snee. "Lean Six Sigma - getting better all the time”. Int. J. Lean Six Sigma. Vol. 1
$\mathrm{N}^{\circ}$ 1, pp. 9-29. 2010. ISSN: 2040-4166. DOI: $10.1108 / 20401461011033130$.

[9] M. Vijaya Sunder. "Lean Six Sigma in higher education institutions". Int. J. Qual. Serv. Sci. Vol. $8 \mathrm{~N}^{\circ} 2$, pp. 159-178. 2016. ISSN: 1756669X. DOI: 10.1108/IJQSS-04-2015-0043.

[10] P. Ramasubramanian. "Six Sigma in Educational Institutions". Int. J. Eng. Pract. Res. Vol. $1 \mathrm{~N}^{\circ} 1$, pp. 1-5. 2012. ISSN: 2326-5914.

[11] Q.H. Mazumder. "Applying Six Sigma in Higher Education Quality Improvement". In 121st ASEE Annual Conference \& Exposition. 2014.

[12] K.G.D. Prasad, K.V. Subbaiah and G. Padmavathi. "Application of six sigma methodology in an engineering educational institution". Int. J. Emerg. Sci. Vol. $2 \mathrm{~N}^{\circ} 2$, pp. 222-237. 2012. ISSN: 2222-4254.

[13] D.M. Arango and B.E. Angel. "Plan de implementación de six Sigma en el proceso de admisiones de una institución de educación superior". Vol. $10 \mathrm{~N}^{\mathrm{o}}$ 2, pp. 13-21. 2012. ISSN: 1692-8261.

[14] M.C. Holmes, A. Kumar and L.O. Jenicke. "Improving the effectiveness of the academic delivery process utilizing Six Sigma". Issues Inf. Syst. Vol. $1 \mathrm{~N}^{\circ}$ 4, p. 15. 2005. ISSN: 1529-7314.

[15] T. Waterbury. "Learning from the pioneers". Int. J. Qual. Reliab. Manag. Vol. 32 N $^{\circ}$ 9, pp. 934-950. 2015. ISSN: 0265-671X. DOI: 10.1108/IJQRM-08-2014-0125.

[16] N. Simons. "The Business Case for Lean Six Sigma in Higher Education". ASQ High. Educ. Briefigher Educ. Br. Vol. $6 \mathrm{~N}^{\mathrm{o}} 3$, pp. 1-6. 2013. ISSN: 2161-265X.

[17] M. Iqbal Khan, S. M. Mourad and W. M. Zahid. "Developing and qualifying Civil Engineering Programs for ABET accreditation". J. King Saud Univ. - Eng. Sci. Vol. $28 \mathrm{~N}^{\circ}$ 1, pp. 1-11. 2016. ISSN: 10183639. DOI: 10.1016/j.jksues.2014.09.001.

[18] J. Lu, C. Laux and J. Antony. "Lean Six Sigma leadership in higher education institutions". Int. J. Product. Perform. Manag. Vol. 66 No 5. 2017. ISSN: 1741-0401. DOI: 10.1108/ IJPPM-09-2016-0195.

[19] L.O. Jenicke, A. Kumar and M.C. Holmes. "A framework for applying six sigma improvement methodology in an academic 
environment". TQM Mag., 2008. ISSN: 09574093. DOI: 10.1108/MBE-09-2016-0047.

[20] J. Antony. "Challenges in the deployment of LSS in the higher education sector," Int. J. Product. Perform. Manag. Vol. $64 \mathrm{~N}^{\circ} 6$, pp. 893-899. 2015. ISSN: 1741-0401. DOI: 10.1108/IJPPM-12-2014-0192.

[21] W.K. Balzer, M.H. Brodke and E.T. Kizhakethalackal. "Lean higher education: successes, challenges, and realizing potential". Meas. Bus. Excell. Vol. $21 \mathrm{~N}^{\circ}$ 2, pp. 191206. 2017. ISSN: 1368-3047. DOI: 10.1108/ MBE-09-2016-0047.

[22] M.P. Sarria Yepess, G.A. Fonseca Villamarin and C.C. Bocanegra-herrera. "Modelo metodológico de implementación de Lean Manufacturing". Esc. Adm. y Negocios. No 83, pp. 51-71. 2017. ISSN: 0120-8160. DOI: 10.21158/01208160.n83.2017.1825.

[23] K. Linderman, R.G. Schroeder, S. Zaheer and A.S. Choo. "Six Sigma: A goal-theoretic perspective". J. Oper. Manag. Vol. $21 \mathrm{~N}^{\circ} 2$, pp. 193-203. 2003. ISSN: 02726963. DOI: 10.1016/S0272-6963(02)00087-6.

[24] J. Burtner. "The Adaptation of Six Sigma Methodology to the Engineering Education Enterprise". In ASEE Southeast Section Conference. 2004.

[25] I.P. M. Barroso, S.M.F. Santos and M.A. Carravilla. "Beyond classroom boundaries: How higher education institutions apply LEAN". 1st Braziliar Symp. Serv. Sci. No 1990. 2010.

[26] J.K. Bandyopadhyay and R. Lichman. "Six Sigma Approach To Quality and Productivity". Vol. $24 \mathrm{~N}^{\circ}$ 4, pp. 1-5. 2007.

[27] M.G. Pryor, C. Alexander and S. Taneja. "The application of Six Sigma methodologies to university processes: The use of student teams ms". J. Case Stud. Accredit. Assess., pp. 1-14. 2012. ISSN: 1941-3386.

[28] A. Kukreja, J.M. Ricks and J.A. Meyer. "Using Six Sigma for performance improvement in business curriculum: A case study". Perform. Improv. Vol. $48 \mathrm{~N}^{\circ}$ 2, pp. 9-25, 2009. ISSN: 10908811. DOI: 10.1002/pfi.20042.

[29] L. Gonzalez. "Desarrollo de un Modelo Seis Sigma para su Implementación en Instituciones de Educación Superior”. 2007. ISBN: 9789586913669.

[30] M. de E. Nacional. "Deserción estudiantil en la educación superior colombiana”. Bogota. 2009.
[31] S.K. Hargrove and L. Burge. "Developing a six sigma methodology for improving retention in engineering education". 32nd Annu. Front. Educ. Vol. 3, pp. 20-24. 2002.

[32] J. Antony. "Six Sigma vs Lean". Int. J. Product. Perform. Manag. Vol. 60, pp. 185-190. 2011. ISSN: 1741-0401. DOI: 10.1108/17410401111101494.

[33] G. Yadav and T.N. Desai. "Lean Six Sigma: a categorized review of the literature". Int. J. Lean Six Sigma. Vol. 7 N$^{\circ}$ 1, pp. 2-24. 2016. ISSN: 2040-4166. DOI: $10.1108 /$ IJLSS-05-2015-0015. ISSN: 2040-4166. DOI: 10.1108/IJLSS-05-2015-0015.

[34] J.P. Womack and D.T. Jones. "Lean ThinkingBANISH WASTE AND CREATE WEALTH IN YOUR CORPORATION". N ${ }^{\circ}$ 1. 1996. Free Press. ISBN: 978-0743231640.

[35] J.A. Douglas, J. Antony and A. Douglas. "Waste identification and elimination in HEIs: the role of Lean thinking". Int. J. Qual. Reliab. Manag. Vol. 32 № 9, pp. 970-981. 2015. ISSN: 0265-671X. DOI: 10.1108/ IJQRM-10-2014-0160.

[36] L. Tatikonda. "Applying Lean Principles to Design, Teach, and Assess Courses". Spring. Vol. 8 No 3, pp. 27-38. 2007.

[37] W. Balzer. "Lean higher education”. 2014. Taylor \& Francis Group. New York, p. 292. 2014. ISBN: 9781439814666.

[38] P.S. Kang and L.M. Manyonge. "Exploration of Lean Principals in Higher Educational Institutes - Based on Degree of Implementation and Indigence". Int. J. Sci. Eng. Res. Vol. 5 $\mathrm{N}^{\circ}$ 2. 2014. ISSN: 2229-5518.

[39] M. Jahan and A. Doggett. "A Study on the Students' Perceptions of the Applicability of Lean Principles at Universities". 2015 ASEE Annu. Conf. Expo. Proc., p. 26.115.126.115.17. 2015.

[40] C.L. Comm and D.F.X. Mathaisel. "An exploratory study of best lean sustainability practices in higher education". Qual. Assur. Educ. Vol. 13 N $^{\circ}$ 3, pp. 227-240. 2005. ISSN: 0968-4883. DOI: 0.1108/09684880510607963

[41] M.L. Emiliani. "Improving business school courses by applying lean principles and practices". Qual. Assur. Educ. Vol. $12 \mathrm{~N}^{\circ} 4$, pp. 175-187. 2004. ISSN: 0968-4883. DOI: $10.1108 / 09684880410561596$. 
[42] M.L. Emiliani. "Using kaizen to improve graduate business school degree programs". Qual. Assur. Educ. Vol. 13 № 1, pp. 37-52. 2005. ISSN: 0968-4883. DOI: $10.1108 / 09684880510578641$.

[43] P. Hines and S. Lethbridge. "New Development: Creating a Lean University". J. Compil. $\mathrm{N}^{\circ}$ February, pp. 53-56. 2008. ISSN: 0954-0962.

[44] A. Thomas, J. Antony, C. Haven-Tang, M. Francis and R. Fisher. "Implementing Lean Six Sigma into curriculum design and delivery - a case study in higher education". Vol. $66 \mathrm{~N}^{\circ}$ 5. 2017. ISSN: 1741-0401. DOI: 10.1108/IJPPM-08-2016-0176.

[45] J. Robert et al. "Propuesta metodológica para la identificación del valor agregado como input de Lean Services en instituciones de educación superior". Rev. Ing. Ind. Vol. 32, pp. 91-115. 2014. ISSN: 1025-9929. DOI: 10.26439/ing.ind2014.n032.117

[46] S. Souraj, A. Rahim and J. Carretero. "The integration of Six Sigma and lean management". Int. J. Lean Six Sigma. Vol. $1 \mathrm{~N}^{\circ} 3$, 2010. ISSN: 2040-4166. DOI: 10.1108/20401461011075035.

[47] E.A. Cudney, S.S.J. Venuthurumilli, T. Materla and J. Antony. "Systematic review of Lean and Six Sigma approaches in higher education". Total Qual. Manag. Bus. Excell. Vol. 0 N $^{\circ}$ 0, pp. 1-14, 2018. ISSN: 14783371. DOI: 10.1080/14783363.2017.1422977.

[48] M. Vijaya Sunder. "Constructs of quality in Higher Education services". Int. J. Product. Perform. Manag. Vol. $60 \mathrm{~N}^{\circ}$ 6, pp. 6-21, 2016. ISSN: 1741-0401. DOI: 10.1108/ IJPPM-05-2015-0079.

[49] J.D. Hess and B.A. Benjamin. "Applying Lean Six Sigma within the university: opportunities for process improvement and cultural change". Int. J. Lean Six Sigma. Vol. 6 $\mathrm{N}^{\circ} 3$, pp. 249-262, 2015. ISSN: 2040-4166. DOI: 10.1108/IJLSS-12-2014-0036.

[50] M.A. Badri, D. Davis, D. Davis, D. Davis and D. Davis. "Lean Six Sigma Journey in a UK Higher Education Institute: A Case Study”. Int. J. Qual. Reliab. Manag. Vol. 35 No 2, pp. 460-471, 2018. ISBN: 0265671981. DOI: 10.1108/IJQRM-01-2017-0005.

[51] S.K. Gupta, J. Antony, F. Lacher and J. Douglas. "Lean Six Sigma for reducing student dropouts in higher education - an exploratory study". Total Qual. Manag. Bus. Excell. Vol. 0 $\mathrm{N}^{\circ} 0$, pp. 1-16, 2018. ISSN: 14783371. DOI: 10.1080/14783363.2017.1422710.

[52] J. Antoyny, C. Madras, and M. Vijaya Sunder. "A Conceptual Lean Six Sigma framework for Quality Excellence in Higher Education Institutions". Int. J. Qual. Reliab. Manag. Vol. $35 \mathrm{~N}^{\circ}$ 4, 2018. ISSN: 0265-671X. DOI: 10.1108/IJQRM-01-2017-0002.

[53] G. Narayanamurthy, A. Gurumurthy, and R. Chockalingam. "Applying lean thinking in an educational institute - an action research". Int. J. Product. Perform. Manag. Vol. $66 \mathrm{~N}^{\circ} 5$, pp. 598-629, 2017. ISSN: 1741-0401. DOI: 10.1108/IJPPM-07-2016-0144.

[54] C. Laux, J. Springer, C. Seliger and N. Li. "Impacting big data analytics in higher education through Six Sigma techniques". Int. J. Product. Perform. Manag. Vol. 66 No 5, 2017. ISSN: 1741-0401. DOI: 10.1108/ IJPPM-09-2016-0194.

[55] A. Thomas, J. Antoyny, M. Francis, and R. Fisher. "A comparative study of Lean implementation in higher and further education institutions in the UK". Int. J. Qual. Reliab. Manag. Vol. $25 \mathrm{~N}^{\circ}$ 8, pp. 878-895, 2016. ISSN: 0265-671X. DOI: 10.1108/ IJQRM-09-2014-0134.

[56] E. Thirkell and I. Ashman. "Lean towards learning: connecting Lean Thinking and human resource management in UK higher education". Int. J. Hum. Resour. Manag. Vol. $25 \mathrm{~N}^{\circ} 21$, SI, pp. 2957-2977, 2014. ISSN: 0958-5192. DOI: 10.1080/09585192.2014.948901.

[57] L. Zhao. "China's higher education quality management based on Six-Sigma management principles". 2011. 2nd Int. Conf. Artif. Intell. Manag. Sci. Electron. Commer, pp. 65596561, 2011.

[58] M.S. Doman. "A new lean paradigm in higher education: a case study". Qual. Assur. Educ. Vol. $19 \mathrm{~N}^{\circ} 3$, pp. 248-262. 2011. ISSN: 09684883. DOI: $10.1108 / 09684881111158054$.

[59] R. Raifsnider and D. Kurt. "Lean six sigma in higher education: Applying proven methodologies to improve quality, remove waste, and quantify opportunities in colleges and universities". White Pap., pp. 1-10. 2004.

[60] Seamus O'Reilly, J. Healy and R. O'Dubhghaill. "Continuous improvement 
in a university - the first steps: a reflective case study". Int. J. Product. Perform. Manag. 2018. ISBN: 0144357011. DOI: 10.1108/ IJPPM-08-2016-0179.

[61] V.G.I.V. Duro Novoa. "La competitividad en las instituciones de educación superior. Aplicación de filosofías de gestión empresarial: LEAN, SIX SIGMA y BUSINESS PROCESS MANAGEMENT (BPM)". Med. (Buenos Aires). Vol. $76 \mathrm{~N}^{\circ}$ 2, pp. 166-181. 2016. ISSN: 0025-7680. DOI: 10.18271/ria.2016.197.

[62] V. Wiegel and L.B. Hadzialic. "Lessons from higher education: adapting Lean Six Sigma to account for structural differences in application domains". Int. J. Six Sigma Compet. Advant. Vol. $9 \mathrm{~N}^{\circ}$ 1, p. 72. 2015. ISSN: 1479-2494. DOI: 10.1504/ IJSSCA.2015.070104.

[63] M.C. Holmes, L.O. Jenicke and J.L. Hempel. "A framework for Six Sigma project selection in higher educational institutions, using a weighted scorecard approach". Qual. Assur. Educ. Vol. 23 No 1, pp. 30-46. 2015.

[64] A. Al Kuwaiti and A.V. Subbarayalu. "Appraisal of students experience survey (SES) as a measure to manage the quality of higher education in the Kingdom of Saudi Arabia: an institutional study using six sigma model". Educ. Stud. Vol. $41 \mathrm{~N}^{\circ} 4$, pp. 430-443. 2015. ISSN: 14653400 . DOI: 10.1080/03055698.2015.1043977.

[65] K. Mukhopadhyay. "Application of Lean six sigma in Indian higher education system". 2017 Int. Conf. Innov. Mech. Ind. Appl. Icimia, pp. 519-523, 2017. ISBN: 978-1-5090-59607. DOI: 10.1109/ICIMIA.2017.7975513.

[66] C. Svensson, J. Antony, M. Ba-Essa, M. Bakhsh and S. Albliwi. "A Lean Six Sigma program in higher education". Int. J. Qual. Reliab. Manag. Vol. $32 \mathrm{~N}^{\circ}$ 9, pp. 951-969, 2015. ISSN: 0265-671X. DOI: 10.1108/ IJQRM-09-2014-0141.

[67] J. Antony. "Readiness factors for the Lean Six Sigma journey in the higher education sector". Int. J. Product. Perform. Manag. Vol. $63 \mathrm{~N}^{\circ} 2$, pp. 257-264, 2014. ISSN: 17410401. DOI: 10.1108/IJPPM-04-2013-0077.

[68] C.L. Comm and D.F.X. Mathaisel. "A case study in applying lean sustainability concepts to universities," Int. J. Sustain. High. Educ. Vol. $6 \mathrm{~N}^{\circ} 2$, pp. 134-146, 2005. ISSN: 14676370. DOI: $10.1108 / 14676370510589855$. 